\title{
A facile and general approach for the synthesis of fluorescent silica nanoparticles doped with inert dyes
}

\author{
MU Xue, WU ChuanLiu, LAI JinPing, CHEN JianBin, ZHENG JinSheng, LI Chong \& \\ ZHAO YiBing*
}

Department of Chemistry and the Key Laboratory of Analytical Sciences of Xiamen University, College of Chemistry and Chemical Engineering, Xiamen University, Xiamen 361005, China

Received April 22, 2011; accepted July 28, 2011

\begin{abstract}
A general and facile approach was developed for the synthesis of almost monodisperse fluorescent silica nanoparticles (NPs) doped with inert dyes, which are organic fluorophores that are strongly fluorescent but are hydrophobic or lack a covalent binding group. The prepared NPs were mesoporous and the dye molecules were encapsulated in the pores via hydrophobic interaction with the CTAB template. The NPs were stable and highly fluorescent in aqueous solution, and have potential applications in bioanalysis and fluorescence encoding.
\end{abstract}

fluorescent probe, silica nanoparticles, encoded microsphere

Citation: $\quad \mathrm{Mu} \mathrm{X}, \mathrm{Wu}$ C L, Lai J P, et al. A facile and general approach for the synthesis of fluorescent silica nanoparticles doped with inert dyes. Chinese Sci Bull, 2011, 56: 3242-3246, doi: 10.1007/s11434-011-4727-1

Because of their unique chemical and optical properties and potential applications in bioimaging, labeling, clinical diagnosis and drug delivery, fluorescent nanoparticles (NPs) have attracted much attention [1-8]. Among the numerous fluorescent NPs, dye-doped silica NPs prepared by incorporating organic dyes into nano- or microsized silica spheres are the most common. These NPs have considerable advantages over conventional molecular dyes because they combine the desirable optical properties of the organic dye with the convenience of a solid material. Embedding a large number of dye units into the silica matrix leads to an intense fluorescence signal that can be $10^{5}$ times that of a single organic fluorophore [9]. The bright fluorescence of these silica NPs makes them especially suitable for ultrasensitive bioanalysis and bioimaging. The silica matrix serves as a shell that protects the fluorophore from diffusion quenching by species such as oxygen. Moreover, the silica matrix is easy to modify and non-cytotoxic, which makes the dye doped silica NPs suitable for biological applications $[10,11]$.

*Corresponding author (email: ybzhao@xmu.edu.cn)
A variety of fluorophores such as fluorescein isothiocyanate, rhodamine isothiocyanate, and tris(2,2'-bipyridine) ruthenium have been successfully incorporated into the si lica matrix by the Stöber method or reversible microemulsion processes to produce fluorescent silica NPs[4,12-16]. However, these methods are limited to dyes that are covalently modified or positively charged because the dye molecules are incorporated into the silica matrix by covalent binding or electrostatic attraction. Unfortunately, most of the highly fluorescent and photostable fluorophores that are currently used, such as pyrene, fluorescein and coumarin, are hydrophobic or lack modifiable groups, and these are referred to as inert dyes in this work. Consequently, directly utilization of these inert dyes in bioanalysis or incorporation into silica NPs by the existing methods is limited. Trapping of the inert dyes into silica NPs would greatly extend the range of usable organic dyes for preparation of fluorescent NPs. However, few studies have investigated the preparation of fluorescent silica NPs doped with inert dyes [17-19].

Herein we propose a facile approach for the preparation of almost monodisperse and highly fluorescent silica 
NPs doped with inert dyes such as pyrene, fluorescein, rhodamine B, and 1,2,3,4,5-pentaphenyl-1,3-cyclopentadiene (PPCP). The prepared silica NPs are mesoporous with pore diameters of several hundred nanometers, and the inert dyes are encapsulated in these pores by hydrophobic interaction with the template molecule, cetyltrimethylammonium bromide (CTAB). Existing methods utilize hydrophobic silicate precursors to trap hydrophobic fluorophores [17-19]. In comparison to these methods, the procedures reported here is shorter, more efficient, and does not require any specific silicate reagent. This method is suitable as a general method for the preparation of inert dye-doped fluorescent silica NPs.

\section{Experimental}

\subsection{Materials and apparatus}

Pyrene, fluorescein, rhodamine B, PPCP, CTAB, tetraethyl orthosilicate (TEOS) and ammonium hydroxide (mass fraction $28 \%, \mathrm{NH}_{3}$ in water) were purchased from J\&K Scientific Ltd., Beijing, China. All other chemicals and solvents are commercially available. Fluorescence spectra were recorded on a RF-5301pc (Shimadzu, Kyoto, Japan) spectrofluorometer, and absorption spectra on a U3900H (Hitachi, Tokyo, Japan) spectrophotometer. Scanning electron microscopy (SEM) micrographs were recorded using a LEO1530 scanning electron microscope (Carl Zeiss NTS $\mathrm{GmbH}$, Oberkochen, Germany). High resolution transmission electron microscopy was performed on a Tecnai F30 transmission electron microscope (FEI, Hillsboro, OR). X-ray diffraction (XRD) patterns were recorded using a PANalytical X'pert PRO (Almelo, The Netherlands) diffractometer. Fluorescence images were taken with a Leica DMLS (Solms, Germany) fluorescence biological microscope coupled with a commercial Canon camera (Tokyo, Japan). Nitrogen adsorption-desorption measurements were performed on a Micromeritics Tristar3000 (Norcross, GA) surface area analyzer at $-196^{\circ} \mathrm{C}$. The $\mathrm{SiO}_{2}$ particles were dried at $200^{\circ} \mathrm{C}$ for $3 \mathrm{~h}$ before analysis. The BurnauerEmmett-Teller (BET) specific surface areas were calculated using the first 10 experimental data points. Pore volumes were determined from the amount of $\mathrm{N}_{2}$ adsorbed at the single point $P / P_{0}=0.98$.

\subsection{Synthesis of dye-doped silica NPs}

Pyrene-doped silica NPs were prepared using a one-step synthesis at room temperature. Pyrene $(0.2 \mathrm{~g})$ was dissolved in $100 \mathrm{~mL}$ of ethanol to prepare a stock solution with a concentration of $0.01 \mathrm{~mol} \mathrm{~L}^{-1}$. Ethanol $(8 \mathrm{~mL})$ and deionized water $(5 \mathrm{~mL})$ were mixed in a $25-\mathrm{mL}$ round-bottom flask and $0.0157 \mathrm{~g}$ of CTAB was added. An aliquot of pyrene stock solution was added to this mixture under stirring. Ammonium hydroxide $(665 \mu \mathrm{L})$ and TEOS $(80 \mu \mathrm{L})$ were then added. The mixture became slightly turbid in $10 \mathrm{~min}$, indicating that hydrolysis of silicate had occurred. After stirring for an additional $4 \mathrm{~h}$, the NPs were isolated from the solution by centrifugation and washed with deionized water several times to remove surfactants and superfluous dye molecules. The obtained NPs were dried in vacuum for $24 \mathrm{~h}$. The NPs prepared by this approach were mesoporous and had a diameter of $600 \mathrm{~nm}$. It is worth noting that the size of the particles could be regulated between $400 \mathrm{~nm}$ and $1.2 \mu \mathrm{m}$ by changing the volume of ammonium hydroxide (see Section 2 for details). Fluorescent silica NPs doped with other inert dyes can be obtained similarly.

\subsection{Determination of dye leakage from dye-doped silica NPs}

The leakage of dye molecules from the pyrene-doped silica NPs in aqueous solution was measured by fluorescence spectrophotometry. The prepared dye-doped silica NPs were dispersed in water and the fluorescence intensity of the solution was measured as $F_{0}$. The solution was then stirred and a $2-\mathrm{mL}$ portion of the solution was taken out and centrifuged at set times. The fluorescence intensity of the upper clear layer was defined as $F_{n}$, and the leakage of the dye from the silica spheres was estimated as $F_{n} / F_{0}$.

\section{Results and discussion}

The inert dye-doped fluorescent silica NPs were prepared by a one-step procedure under homogeneous conditions using water/ethanol binary solvent and a charged surfactant template, CTAB. Smooth spherical particles with ordered mesopores were obtained. SEM, TEM, small-angle XRD and $\mathrm{N}_{2}$ adsorption-desorption measurements were used to characterize the prepared silica NPs. The formation of silica NPs was strongly dependent on the amount of reagents used in the synthesis of the NPs, including ethanol, water, CTAB and ammonium hydroxide. Figure 1(a) and (b) shows the SEM images of the pyrene-doped silica spheres. The NPs were almost monodisperse smooth spheres with diameters around $500 \mathrm{~nm}$. TEM revealed that the particles were mesoporous with pore diameters of approximately 1.0-3.0 nm (Figure 1(c) and (d)). The particle size could be regulated between $400 \mathrm{~nm}$ to $1.2 \mu \mathrm{m}$ by varying the volume of ammonium hydroxide used in the synthesis (Figure 2).

Figure 3(A) shows the small-angle XRD patterns of the prepared silica particles. The NPs with and without pyrene doping exhibited two relatively broad peaks. These strong $\left(2 \theta=2.34^{\circ}\right.$ for curve a, $2.37^{\circ}$ for curve $\left.b\right)$ and weak $(2 \theta=$ $4.48^{\circ}$ for curve $a, 4.44^{\circ}$ for curve b) peaks were characteristic of the (100) and (110) diffraction planes of a hexagonal lattice, respectively [20,21]. These results show that the prepared NPs are mesoporous and that incorporation of pyrene does not influence the structure of the NPs [22]. Furthermore, $\mathrm{N}_{2}$ adsorption-desorption measurements 


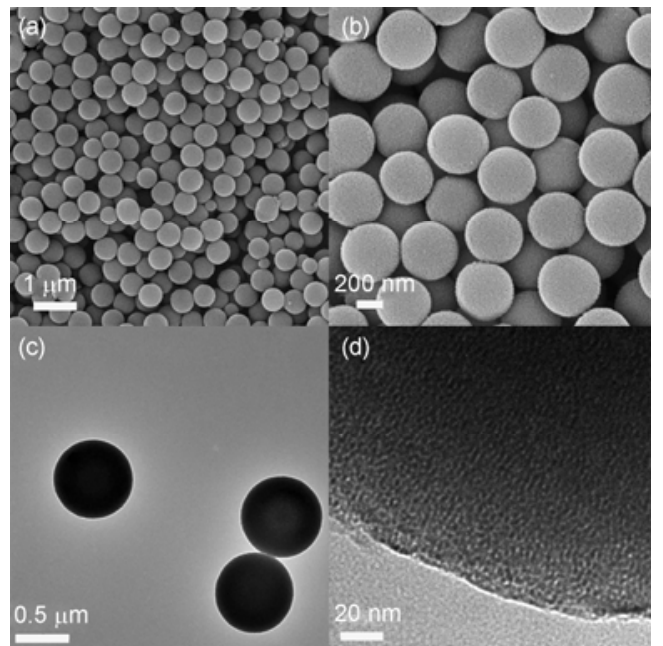

Figure 1 SEM $(a, b)$ and TEM $(c, d)$ characterization of the pyrene-doped silica NPs.

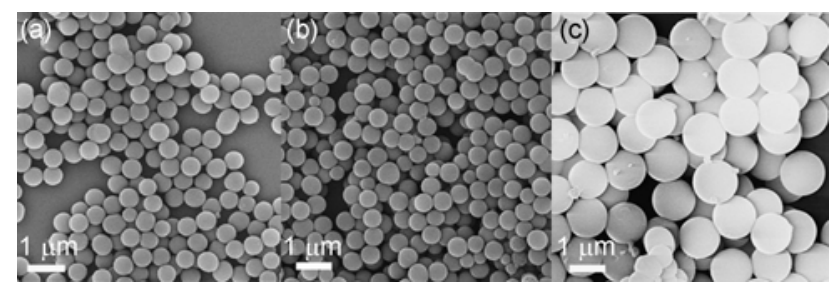

Figure 2 SEM images of pyrene-doped silica NPs prepared using (a) 800, (b) 400 , and (c) $200 \mu \mathrm{L}$ of ammonia hydroxide.
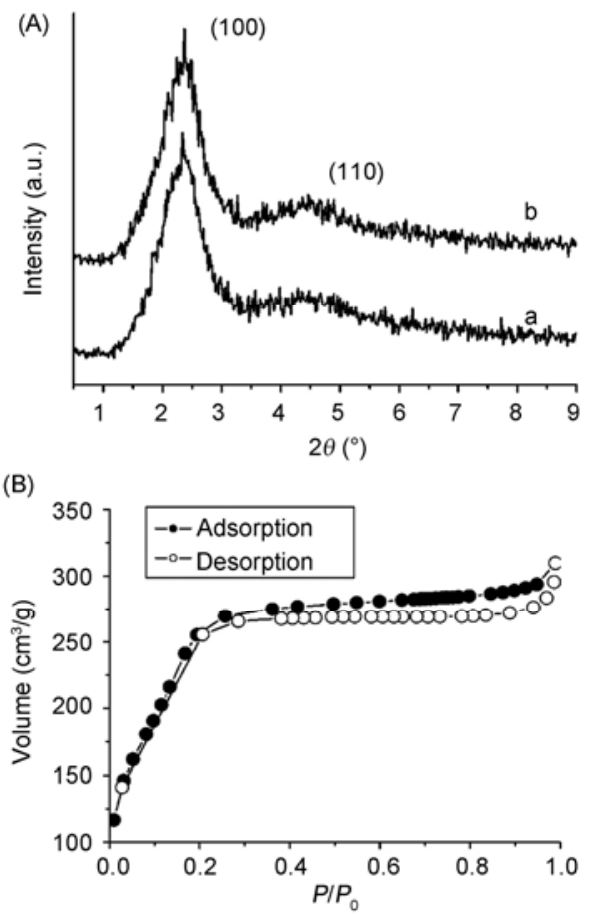

Figure 3 (A) XRD characterization of the silica NPs doped with pyrene (a) and without pyrene (b). (B) $\mathrm{N}_{2}$ adsorption-desorption isotherms of the prepared mesoporous silica NPs.
(Figure 2(b)) of the silica NPs revealed a typical adsorption-desorption isotherm, which indicated the presence of micropores. The BET specific surface area and single-point total pore volume at $P / P_{0}=0.98$ were $925 \mathrm{~m}^{2} / \mathrm{g}$ and 1.09 $\mathrm{cm}^{3} / \mathrm{g}$, respectively. The large pore volume allows the silica NPS to give an intense fluorescence signal because it can accommodate many dye molecules, and this might allow for ultrasensitive bioanalysis and bioimaging.

Absorption spectra and fluorescence spectra of the pyrene-doped silica NPs are shown in Figure 4. The NPs absorption peaks at 242, 275, 323 and $339 \mathrm{~nm}$ were slightly red-shifted compared to those of pyrene in ethanol (Figure 4(A), curve c). Comparing curves a, b and c in Figure 4(A), the absorption spectrum of pyrene-doped silica NPs appears to be a simple overlap of the spectra of the silica NPs and pyrene. The prepared NPs were highly fluorescent, and the fluorescence properties were closely related to the concentration of the fluorophore used in the synthesis. Figure 4(B) shows that the NPs prepared with $1 \mathrm{mmol} \mathrm{L}^{-1}$ pyrene exhibited both the monomer and excimer emission of pyrene (curve a). As the pyrene concentration decreased to $4 \mu \mathrm{mol}$ $\mathrm{L}^{-1}$, the excimer emission disappeared and only the monomer emission was observed. This indicated that the hydrophobic fluorescent dye was successfully embedded into the silica matrix, and that the fluorescence of the NPs could be easily modified by varying the concentration of the dye. Because pyrene does not contain any modifiable groups and is neutral, attaching pyrene to the silica matrix by covalent binding or electrostatic attraction is not feasible. It is therefore reasonable to assume that the hydrophobic dye
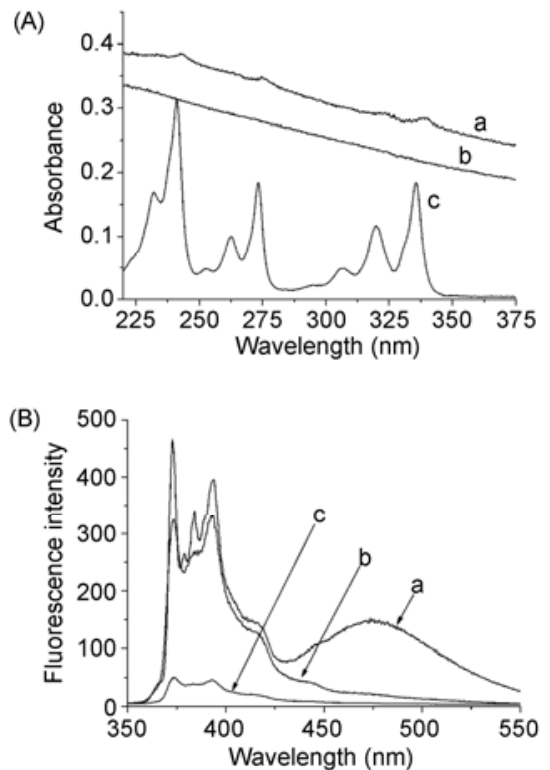

Figure 4 (A) Absorption spectra of silica NPs doped with pyrene (a) or without pyrene (b) and an ethanol solution of pyrene (c). (B) Fluorescence spectra of silica NPs doped with pyrene at concentrations of $1 \mathrm{mmol} \mathrm{L}^{-1}$ (a) and $4 \mu \mathrm{mol} \mathrm{L}^{-1}$ (b), and of the pyrene-doped silica NPs after washing with ethanol (c). 
molecules are loaded into the pores of the silica NPs byhydrophobic interaction with the CTAB template. To verify this assumption, the fluorescent NPs were washed with ethanol to remove the CTAB template and the doped dye. After this, the fluorescence of NPs was dramatically quenched (Figure 4(B), curve c) and the ethanol solution became strongly fluorescent, which confirmed that most of the doped dye was removed from the silica matrix by the ethanol.

CTAB is known to form hexagonal liquid crystals spontaneously at concentrations above its critical micelle concentration (CMC) in homogeneous solutions. During the synthesis procedure, these hexagonal micelles were assumed to serve as the template to form the mesoporous structure of the silica NPs [23-26]. However, the procedure reported here is considered to be silica driven because the concentration of CTAB is below its CMC (for details see Section 2.2) [20,21,27]. As illustrated in Scheme 1, hydrolysis of TEOS first forms negatively charged silica polymers, which strongly interact with the cationic surfactant CTAB to form multidentate links. The resultant stable silica/ surfactant complexes would have very low dissociation constants and CMCs [28]. Thus, the silica driven formation of micelles occurred and the hydrophobic inert dye pyrene was concentrated in the hydrophobic core through hydrophobic interactions. By subsequently hydrolysis and growth, the dye molecules were encapsulated inside the formed silica NPs. This was supported by the absence of NP formation when $\mathrm{CTAB}$ was not included in the synthesis procedure.

The leakage of dye molecules from the NPs is an important parameter for evaluating the stability of the dye-doped silica NPs. The leakage of pyrene molecules from the silica NPs in aqueous solution was $<4 \%$ after dispersion of the pyrene-doped silica NPs in water for one week, which demonstrates that the dye-doped silica NPs are highly stable in aqueous solution. However, almost all the dye leaked out when the NPs were dispersed in organic solvents such as ethanol. To address this problem, the silica particles can be modified by applying a additional stable silica coating (post-coating). The dye-doped silica particles would then be stable in organic or aqueous solutions, and could be further functionalized and bioconjugated with biomolecules for bioanalysis [10,29].

Finally, the hydrophobic dye PPCP and two traditional fluorophores, fluorescein and rhodamine B, were also tested for their incorporation into the silica NPs. Fluorescence images (Figure 5) showed that the doped fluorescent silica NPs were well dispersed and ordered. The fluorescent encoded silica NPs were easily prepared using the method developed in this study. Figure 6 shows the multicolor

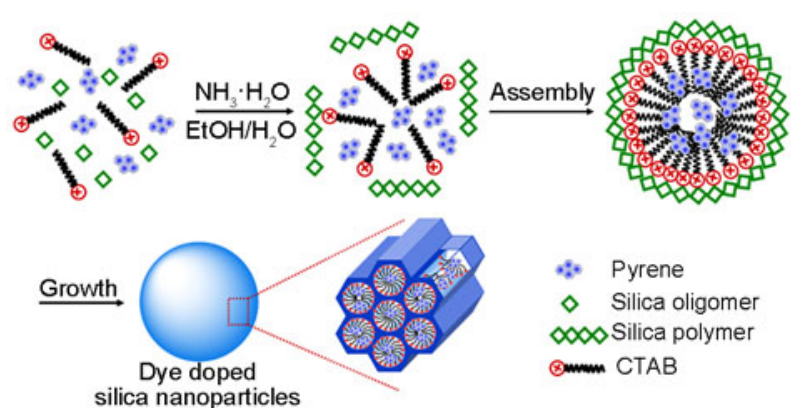

Scheme 1 Preparation of hydrophobic dye-doped silica NPs.
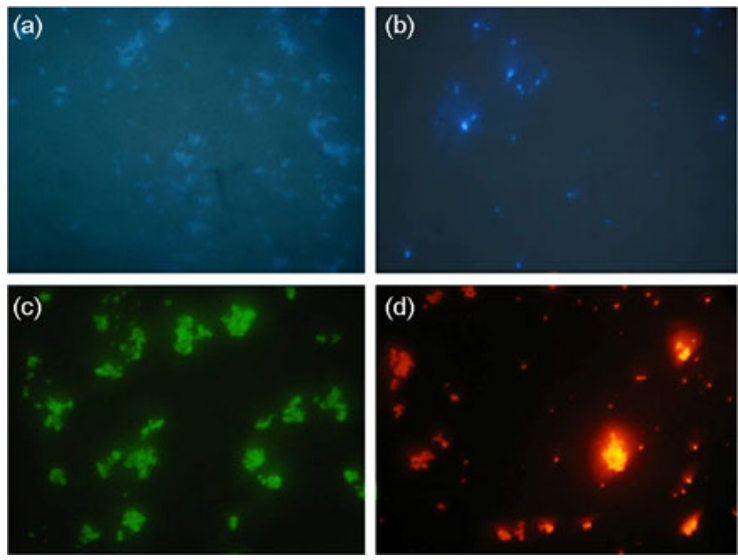

Figure 5 Fluorescence images of fluorescent silica nanoparticles doped with (a) pyrene, (b) PPCP, (c) fluorescein and (d) rhodamine B.

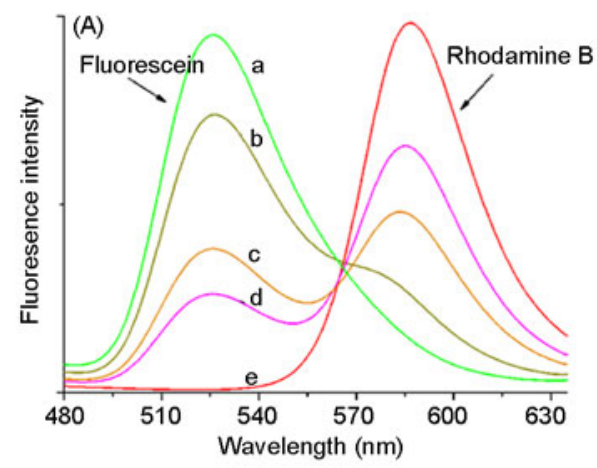

(B)

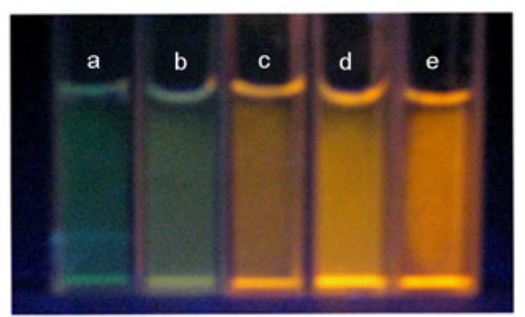

Figure 6 (A) Fluorescence spectra of silica NPs encoded with different amounts of fluorescein and rhodamine B; (B) the corresponding fluorescence imaging under an UV lamp. [Fluorescein] : [Rhodamine B] = 1:0(a), 3:1(b), 2:1(c), 1:1(d) and 0:1(e). 
fluorescence emitted from the silica NPs containing various ratios of fluorescein and rhodamine $\mathrm{B}$.

\section{Conclusions}

In conclusion, a facile approach was developed for the synthesis of almost monodisperse and ordered inert dye-doped fluorescent silica NPs via dye encapsulation. The brightly fluorescent silica NPs were mesoporous and stable in aqueous solution. This general approach will extend the range of organic dyes that can be used in the preparation of the fluorescent NPs, and it offers a facile yet highly efficient route for synthesis of fluorescent NPs for bioanalysis and optoelectronic nanodevices.

This work was supported by the National Natural Science Foundation of China (20875079 and 20835005), the Planned Science and Technology Project of Xiamen, China (3502z20080011) and the Specialized Research Fund for the Doctoral Program of Higher Education of China (200803840007)

1 Lee S J, An B K, Jung S D, et al. Photoswitchable organic nanoparticles and a polymer film employing multifunctional molecules with enhanced fluorescence emission and bistable photochromism. Angew Chem Int Ed, 2004, 43: 6346-6350

2 Fu H B, Yao J N. Size effects on the optical properties of organic nanoparticles. J Am Chem Soc, 2001, 123: 1434-1439

3 Larson D R, Zipfel W R, Williams R M, et al. Water-soluble quantum dots for multiphoton fluorescence imaging in vivo. Science, 2003, 300: 1434-1436

4 Wang L, Wang K, Santra S, et al. Watching silica nanoparticles glow in the biological world. Anal Chem, 2006, 78: 646-654

5 Hasegawa U, Nomura S I, Kual S C, et al. Nanogel-quantum dot hybrid nanoparticles for live cell imaging. Biochem Biophys Res Commun, 2005, 331: 917-921

6 Meldal M. The one-bead two-compound assay for solid phase screening of combinatorial libraries. Biopolymers, 2002, 66: 93-100

7 Wu C, Zheng J, Huang C, et al. Hybrid silica-nanocrystal-organic dye superstructures as post-encoding fluorescent probes. Angew Chem Int Ed, 2007, 46: 5393-5396

8 Kumar R, Roy I, Ohulchanskyy T Y, et al. Covalently dye-linked, surface-controlled, and bioconjugated organically modified silica nanoparticles as targeted probes for optical imaging. ACS Nano, 2008, 2: 449-456

9 Zhao X, Bagwa R P, Tan W. Development of organic-dye-doped silica nanoparticles in a reverse microemulsion. Adv Mater, 2004, 16: 173-176

10 Matsumoto A, Tsutsumi K, Schumacher K, et al. Surface functionalization and stabilization of mesoporous silica spheres by silanization and their adsorption characteristics. Langmuir, 2002, 18: 4014-4019

11 Tan W, Wang K, He X, et al. Bionanotechnology based on silica nanoparticles. Med Res Rev, 2004, 24: 621-638

12 Gao D, Wang Z, Liu B, et al. Resonance energy transfer-amplifying fluorescence quenching at the surface of silica nanoparticles toward ultrasensitive detection of TNT. Anal Chem, 2008, 80: 8545-8553

13 Sokolov I, Kievsky Y Y, Kaszpurenko J M. Self-assembly of ultrabright fluorescent silica particles. Small, 2007, 3: 419-423

14 Muto S, Oaki Y, Imai H. Incorporation of dyes into silica-surfactant mesostructured nanoparticles as a nanoscale host material for organic molecules. Chem Lett, 2006, 35: 880-881

15 Lin Y S, Tsai C P, Huang H Y, et al. Well-ordered mesoporous silica nanoparticles as cell markers. Chem Mater, 2005, 17: 4570-4573

16 Santra S, Zhang P, Wang K, et al. Conjugation of biomolecules with luminophore-doped silica nanoparticles for photostable biomarkers. Anal Chem, 2001, 73: 4988-4993

17 Wang Q, Choy R, Dai Q, et al. Preparation of dye-loaded $\mathrm{SiO}_{2}$ nanoparticles. J Non-Crys Sol, 2007, 353: 354-365

18 Tapec R, Zhao X J, Tan W. Development of organic dye-doped silica nanoparticles for bioanalysis and biosensors. J Nanosci Nanotechnol, 2002, 2: 405-409

19 Shibata S, Taniguchi T, Yano T, et al. Formation of water-soluble dye-doped silica particles. J Sol-Gel Sci Technol, 1997, 10: 263-268

20 Nooney R, Thirunavukkarasu D, Chen Y, et al. Synthesis of nanoscale mesoporous silica spheres with controlled particle size. Chem Mater, 2002, 14: 4721-4728

21 Frasch J, Lebeau B, Soulard M, et al. In situ investigations on cetyltrimethylammonium surfactant/silicate systems, precursors of organized mesoporous MCM-41-Type siliceous materials. Langmuir, 2000, 16: 9049-9057

22 Chen $\mathrm{H}, \mathrm{He} \mathrm{J}$, Tang $\mathrm{H}$, et al. Porous silica nanocapsules and nanospheres: Dynamic self-assembly synthesis and application in controlled release. Chem Mater, 2008, 20: 5894-5900

23 Keresge C T, Leonowicz M Z, Roth W J. Ordered mesoporous molecular sieves synthesized by a liquid-crystal template mechanism. Nature, 1992, 359: 710-712

24 Beck J S, Vartuli J C, Roth W J, et al. A new family of mesoporous molecular sieves prepared with liquid crystal templates. J Am Chem Soc, 1992, 114: 10834-10843

25 Cai Q, Lin W Y, Xiao F S, et al. The preparation of highly ordered MCM-41 with extremely low surfactant concentration. Microporous Mesoporous Mater, 1999, 32: 1-15

26 Li H L, Perkas N, Li Q L, et al. Improved silanization modification of a silica surface and its application to the preparation of a silicasupported polyoxometalate catalyst. Langmuir, 2003, 19: 1040910413

27 Jang J, Oh J H. Facile fabrication of photochromic dye-conducting polymer core-shell nanomaterials and their photoluminescence. Adv Mater, 2003, 15: 977-980

28 Hermanson G T. Bionconjugate Techniques. San Diego: Academic Press, 1996

29 Huo Q, Margolese D I, Ciesla U, et al. Organization of organic mole cules with inorganic molecular species into nanocomposite biphase arrays. Chem Mater, 1994, 6:1176-1191

Open Access This article is distributed under the terms of the Creative Commons Attribution License which permits any use, distribution, and reproduction in any medium, provided the original author(s) and source are credited. 